This document was prepared in conjunction with work accomplished under Contract No. DE-AC09-96SR18500 with the U. S. Department of Energy.

\title{
DISCLAIMER
}

This report was prepared as an account of work sponsored by an agency of the United States Government. Neither the United States Government nor any agency thereof, nor any of their employees, nor any of their contractors, subcontractors or their employees, makes any warranty, express or implied, or assumes any legal liability or responsibility for the accuracy, completeness, or any third party's use or the results of such use of any information, apparatus, product, or process disclosed, or represents that its use would not infringe privately owned rights. Reference herein to any specific commercial product, process, or service by trade name, trademark, manufacturer, or otherwise, does not necessarily constitute or imply its endorsement, recommendation, or favoring by the United States Government or any agency thereof or its contractors or subcontractors. The views and opinions of authors expressed herein do not necessarily state or reflect those of the United States Government or any agency thereof. 
Key Words: Dose Determination Saltstone

Retention: Lifetime

\section{POTENTIAL RADIOLOGICAL DOSES FROM GROUNDWATER CONTAMINATED BY THE SALTSTONE DISPOSAL FACILITY (U)}

\section{G.T. JANNIK}

Issued: February 2005

Westinghouse Savannah River Company Savannah River Site Aiken, SC 29808

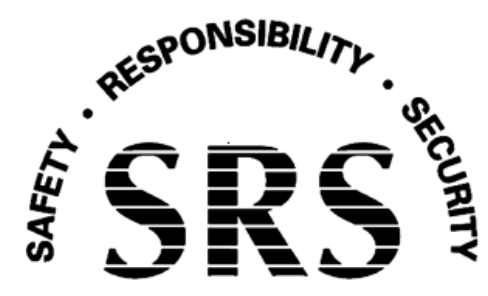


This page was intentionally left blank 


\section{Potential Radiological Doses from Groundwater Contaminated by the Saltstone Disposal Facility (U)}

Assessments of radiological dose from usage of groundwater potentially contaminated by the Saltstone Disposal Facility (Z-Area) were made for a hypothetical future resident farmer. These assessments were made using the routine aqueous release model LADTAP XLC), which is the model used for demonstrating liquid pathway dose compliance at SRS (Simpkins, 2004). The dose factors used in LADTAP XLC) are those specified by the Department of Energy (USDOE, 1988a and 1988b).

It was conservatively assumed that a future resident farmer uses the contaminated groundwater as a source of 1) drinking water, 2) pond water (in which fish are raised and recreational activities occur), and 3) irrigation water used for raising vegetables, meat, and milk.

LADTAP XL ( contains two worksheets: LADTAP and IRRIDOSE. The LADTAP worksheet estimates dose from environmental pathways including external exposure resulting from recreational activities (swimming, boating, and shoreline use) and from ingestion of water and fish. IRRIDOSE estimates dose from food crops irrigated with contaminated water. It was conservatively assumed that all of the food consumed by the resident farmer was irrigated with contaminated groundwater. Therefore, the dose from the irrigation pathway is not a function of the land area irrigated.

The maximally-exposed-individual usage and exposure parameters identified in Simpkins (2004) were used in the calculations.

The 1,000-year and 10,000-year radionuclide concentrations in groundwater, which were calculated by Waste Processing Technology, are provided in Tables 1 and 2. They were used in the dose assessments as follows:

To obtain a particular exposure point concentration in LADTAP XL@, an annual source term $(\mathrm{Ci} / \mathrm{y})$ at a given river flow rate (cfs) must be inputted in the spreadsheet. For this assessment, a 10,000 cfs flow rate was assumed and the radionuclide specific source terms were calculated using the following equation:

$$
Q_{i}=\frac{\bar{C}_{i} * F}{1.12}
$$

where:

$Q_{i} \quad=$ the annual amount of radionuclide i released $(\mathrm{Ci} / \mathrm{y})$

$\bar{C}_{i} \quad=$ the annual average concentration of radionuclide $\mathrm{i}(\mathrm{pCi} / \mathrm{mL})$

$\mathrm{F} \quad=$ the assumed river flow rate (cfs)

$1.12=$ conversion factor $\left(\mathrm{y}-\mathrm{ft}^{3}-\mathrm{pCi} / \mathrm{s}-\mathrm{mL}-\mathrm{Ci}\right)$ 
The estimated doses from each radionuclide, itemized by pathway, are shown in Tables 1 and 2 for the 1,000-year and 10,000-year time periods, respectively.

\section{References}

Simpkins, A.A., LADTAP XLC: A SPREADSHEET FOR ESTIMATING DOSE RESULTING FROM AQUEOUS RELEASES, WSRC-TR-2004-00059, Savannah River Site, Aiken, SC, 2004.

U.S. Department of Energy, External Dose-Rate Conversion Factors for Calculation of Dose to the Public, DOE/EH-0070, Washington, DC 1988a.

U.S. Department of Energy, Internal Dose Conversion Factors for Calculation of Dose to the Public, DOE/EH-0071, Washington, DC, 1988b. 
Table 1. Maximally-Exposed-Individual Doses for a Future Resident Farmer at 1,000 Years

\begin{tabular}{|c|c|c|c|c|c|c|c|c|}
\hline \multirow[b]{2}{*}{ Radionuclide } & \multirow{2}{*}{$\begin{array}{l}\text { Concentration } \\
(\mathrm{pCi} / \mathrm{L})\end{array}$} & \multicolumn{7}{|c|}{$\begin{array}{c}\text { Dose } \\
\text { (mrem/year) }\end{array}$} \\
\hline & & Fish & $\underline{\text { Water }}$ & $\underline{\text { Shoreline }}$ & $\underline{\text { Swimming }}$ & Boating & Crops & Total Dose \\
\hline C-14 & 1.3E-27 & 2.3E-28 & 2.0E-30 & $0.0 \mathrm{E}+00$ & $0.0 \mathrm{E}+00$ & $0.0 \mathrm{E}+00$ & 1.1E-27 & 1.3E-27 \\
\hline $\mathrm{Cl}-36$ & $5.2 \mathrm{E}-25$ & $1.5 \mathrm{E}-27$ & 1.1E-27 & 1.3E-33 & $5.5 E-38$ & $6.5 \mathrm{E}-38$ & $3.2 E-24$ & $3.2 \mathrm{E}-24$ \\
\hline $\mathrm{H}-3$ & $1.4 \mathrm{E}-10$ & $1.5 \mathrm{E}-16$ & $6.2 \mathrm{E}-15$ & $0.0 \mathrm{E}+00$ & 2.7E-18 & $0.0 \mathrm{E}+00$ & 5.4E-15 & $1.2 \mathrm{E}-14$ \\
\hline I-129 & $6.5 \mathrm{E}-09$ & $5.2 \mathrm{E}-10$ & 1.3E-09 & $7.6 \mathrm{E}-12$ & $7.0 \mathrm{E}-16$ & 8.3E-16 & 6.9E-09 & 8.7E-09 \\
\hline $\mathrm{K}-40$ & 3.5E-12 & 1.3E-12 & 4.8E-14 & $2.5 \mathrm{E}-14$ & $6.2 \mathrm{E}-18$ & 7.3E-18 & $1.5 \mathrm{E}-12$ & $2.8 \mathrm{E}-12$ \\
\hline Mo-93 & $6.9 \mathrm{E}-11$ & 1.7E-14 & $6.6 \mathrm{E}-14$ & 2.1E-14 & $6.9 \mathrm{E}-19$ & 8.1E-19 & 8.8E-13 & $9.8 \mathrm{E}-13$ \\
\hline *Nb-93m & $1.0 \mathrm{E}-12$ & $3.2 \mathrm{E}-12$ & 4.0E-15 & $2.4 \mathrm{E}-17$ & $1.9 \mathrm{E}-21$ & $2.2 \mathrm{E}-21$ & $6.6 \mathrm{E}-14$ & $3.2 \mathrm{E}-12$ \\
\hline Sr-90 & $7.9 \mathrm{E}-30$ & 5.9E-31 & 7.5E-31 & $0.0 \mathrm{E}+00$ & $0.0 \mathrm{E}+00$ & $0.0 \mathrm{E}+00$ & $2.8 \mathrm{E}-30$ & $4.1 \mathrm{E}-30$ \\
\hline \multirow[t]{2}{*}{ Tc-99 } & $1.2 \mathrm{E}-21$ & $4.5 \mathrm{E}-25$ & $1.2 \mathrm{E}-24$ & 4.1E-29 & 7.7E-33 & $9.0 \mathrm{E}-33$ & 1.4E-22 & $1.4 \mathrm{E}-22$ \\
\hline & & & & & & & Total $=$ & 8.8E-09 \\
\hline
\end{tabular}


Table 2. Maximally-Exposed-Individual Doses for a Future Resident Farmer at 10,000 Years

\begin{tabular}{|c|c|c|c|c|c|c|c|c|}
\hline \multirow[b]{2}{*}{$\underline{\text { Radionuclide }}$} & \multirow{2}{*}{$\begin{array}{l}\text { Concentration } \\
\quad(\mathrm{pCi} / \mathrm{L}) \\
\text { Groundwater }\end{array}$} & \multicolumn{7}{|c|}{$\begin{array}{c}\text { Dose } \\
\text { (mrem/year) }\end{array}$} \\
\hline & & Fish & $\underline{\text { Water }}$ & $\underline{\text { Shoreline }}$ & Swimming & Boating & Crops & $\underline{\text { Total Dose }}$ \\
\hline Al-26 & $2.1 \mathrm{E}-14$ & 5.3E-17 & $2.0 \mathrm{E}-16$ & 2.8E-15 & $6.8 \mathrm{E}-19$ & 8.0E-19 & $6.4 \mathrm{E}-16$ & 3.7E-15 \\
\hline C-14 & $1.8 \mathrm{E}-21$ & $3.2 \mathrm{E}-22$ & 2.7E-24 & $0.0 \mathrm{E}+00$ & $0.0 \mathrm{E}+00$ & $0.0 \mathrm{E}+00$ & $1.5 \mathrm{E}-21$ & $1.8 \mathrm{E}-21$ \\
\hline $\mathrm{Cl}-36$ & 1.4E-20 & $3.9 \mathrm{E}-23$ & 3.0E-23 & 3.4E-29 & $1.4 \mathrm{E}-33$ & 1.7E-33 & 8.3E-20 & 8.3E-20 \\
\hline Cs-135 & $1.1 \mathrm{E}-11$ & $4.5 \mathrm{E}-12$ & 5.7E-14 & $0.0 \mathrm{E}+00$ & $0.0 \mathrm{E}+00$ & $0.0 \mathrm{E}+00$ & 3.1E-13 & $4.9 \mathrm{E}-12$ \\
\hline H-3 & $1.4 \mathrm{E}-10$ & $1.5 \mathrm{E}-16$ & $6.2 \mathrm{E}-15$ & $0.0 \mathrm{E}+00$ & 2.7E-18 & $0.0 \mathrm{E}+00$ & $5.4 \mathrm{E}-15$ & $1.2 \mathrm{E}-14$ \\
\hline |-129 & 1.6E-04 & 1.3E-05 & 3.2E-05 & $1.8 \mathrm{E}-07$ & $1.7 \mathrm{E}-11$ & $2.0 \mathrm{E}-11$ & 1.7E-04 & $2.2 \mathrm{E}-04$ \\
\hline K-40 & 7.7E-05 & 2.8E-05 & 1.1E-06 & 5.6E-07 & $1.4 \mathrm{E}-10$ & $1.6 \mathrm{E}-10$ & 3.2E-05 & $6.2 \mathrm{E}-05$ \\
\hline Mo-93 & $1.2 \mathrm{E}-04$ & $2.9 \mathrm{E}-08$ & $1.1 \mathrm{E}-07$ & 3.6E-08 & $1.2 \mathrm{E}-12$ & $1.4 \mathrm{E}-12$ & $1.5 \mathrm{E}-06$ & 1.7E-06 \\
\hline *Nb-93m & 2.4E-06 & 7.2E-06 & $9.2 \mathrm{E}-09$ & $5.6 \mathrm{E}-11$ & $4.2 \mathrm{E}-15$ & $5.0 \mathrm{E}-15$ & $1.5 \mathrm{E}-07$ & 7.4E-06 \\
\hline Np-237 & $2.5 \mathrm{E}-21$ & $1.8 \mathrm{E}-21$ & $7.1 \mathrm{E}-21$ & 4.3E-24 & $6.8 \mathrm{E}-28$ & 8.0E-28 & $2.2 \mathrm{E}-20$ & $3.1 \mathrm{E}-20$ \\
\hline **Np-237 & $2.1 \mathrm{E}-25$ & $1.6 \mathrm{E}-25$ & $6.1 \mathrm{E}-25$ & 3.7E-28 & $5.8 \mathrm{E}-32$ & $6.9 \mathrm{E}-32$ & $1.9 \mathrm{E}-24$ & $2.6 \mathrm{E}-24$ \\
\hline Pd-107 & $5.5 E-20$ & $1.5 \mathrm{E}-24$ & $5.6 \mathrm{E}-24$ & $0.0 \mathrm{E}+00$ & $0.0 \mathrm{E}+00$ & $0.0 \mathrm{E}+00$ & $5.8 \mathrm{E}-23$ & $6.5 \mathrm{E}-23$ \\
\hline Rb-87 & $1.1 \mathrm{E}-18$ & 2.0E-19 & $3.8 \mathrm{E}-21$ & $0.0 \mathrm{E}+00$ & $0.0 \mathrm{E}+00$ & $0.0 \mathrm{E}+00$ & $9.9 \mathrm{E}-20$ & 3.0E-19 \\
\hline Se-79 & 2.2E-05 & $5.9 \mathrm{E}-07$ & 1.3E-07 & $0.0 \mathrm{E}+00$ & $0.0 \mathrm{E}+00$ & $0.0 \mathrm{E}+00$ & 2.9E-05 & 3.0E-05 \\
\hline Sr-90 & 8.5E-30 & 6.3E-31 & 8.0E-31 & $0.0 \mathrm{E}+00$ & $0.0 \mathrm{E}+00$ & $0.0 \mathrm{E}+00$ & $3.0 \mathrm{E}-30$ & 4.4E-30 \\
\hline \multirow[t]{2}{*}{ Tc-99 } & $3.9 \mathrm{E}-17$ & $1.4 \mathrm{E}-20$ & 3.7E-20 & $1.3 \mathrm{E}-24$ & $2.4 \mathrm{E}-28$ & $2.9 \mathrm{E}-28$ & $4.5 \mathrm{E}-18$ & $4.6 \mathrm{E}-18$ \\
\hline & & & & & & & Total = & $3.2 \mathrm{E}-04$ \\
\hline
\end{tabular}

${ }^{*} \mathrm{Nb}-93 \mathrm{~m}$ effects as a daughter of Mo-93

**Np-237 effects as a daughter of the primary radionuclide Cf-249 